\title{
Voxel-based analysis of diffusion tensor imaging in patients with mesial temporal lobe epilepsy
}

\author{
Patrícia Sanches $^{a, b}$, Elaine Keiko Fujisao ${ }^{a}$, Aline M.S. Braga ${ }^{a}$, Nathalia Raquel Cristaldo ${ }^{a}$, \\ Roberto dos Reis $^{\mathrm{a}, \mathrm{b}}$, Seizo Yamashita ${ }^{\mathrm{b}}$, Luiz Eduardo Betting ${ }^{\mathrm{a}, *}$ \\ a Departamento de Neurologia, Psicologia e Psiquiatria, Faculdade de Medicina de Botucatu - UNESP - Univ Estadual Paulista, Brazil \\ b Departamento de Doenças Tropicais e Diagnóstico por Imagem, Faculdade de Medicina de Botucatu - UNESP - Univ Estadual Paulista, Brazil
}

\section{A R T I C L E I N F O}

\section{Article history:}

Received 14 May 2016

Received in revised form 19 February 2017

Accepted 24 March 2017

Available online 25 March 2017

\section{Keywords:}

Temporal lobe epilepsy

Hippocampal sclerosis

Neuroimaging

Diffusion tensor imaging

\begin{abstract}
A B S T R A C T
Purpose: Quantitative techniques of diffusion analysis allow for an in-vivo investigation of the physiopathology of epilepsies. The objective of this study was to evaluate the variation of the main diffusion parameters and explore differences between two methodologies of voxel-wise analysis comparing a group of patients with mesial temporal lobe epilepsy (MTLE) with controls.

Methods: 24 patients with a diagnosis of MTLE were selected. All patients and a control group of 36 individuals were submitted to $3 \mathrm{~T}$ magnetic resonance imaging. Diffusion parameters were obtained from the raw images. Based on the tensors, a customized template was created, and images were registered into standard space. Voxel-based comparisons between patients and controls was performed by whole brain voxel-wise analysis and tract-based spatial statistics (TBSS). Tract-specific analysis (TSA) was performed in the mostly damaged fasciculi.

Results: 10 patients presented with right hippocampal sclerosis (HS), 11 with left HS and 3 with bilateral HS with left predominance. Whole brain voxel-wise analysis showed abnormalities mainly localized in the temporal lobes (total volume of $3859 \mathrm{~mm}^{3}$ ). TBSS showed more widespread abnormalities $\left(21931 \mathrm{~mm}^{3}\right)$. TSA pointed to abnormalities situated essentially in the temporal stem topography. Fractional anisotropy (FA) and radial diffusivity (RD) were the parameters that showed more abnormalities. Conclusion: Whole brain voxel-wise analysis was more restricted than TBSS. The methods were complementary stressing the significance of the findings. The abnormalities were more frequently observed in FA and RD indicating the need for using several diffusion parameters for the investigation of patients with MTLE.
\end{abstract}

() 2017 Elsevier B.V. All rights reserved.

\section{Introduction}

Investigations of the white matter in patients with mesial temporal lobe epilepsy (MTLE) using diffusion tensor imaging (DTI) have been able to detect abnormalities predominantly, but unrestricted to, the temporal lobe allocated in a centrifugal pattern (Concha et al., 2012). This pattern is under investigation and changes according to the heterogeneity of the patients and the different methods of neuroimaging analysis, including the acquisition parameters and post-processing algorithms.

Voxel-based analyses are quantitative techniques which have as major advantages the ability to statistically compare groups of brains (Yasuda et al., 2010). In diffusion images, voxel-based anal-

\footnotetext{
* Corresponding author at: Departamento de Neurologia, Psicologia e Psiquiatria, Faculdade de Medicina de Botucatu - UNESP - Univ Estadual Paulista, Zip Code 18618-687, Botucatu, SP, Brazil.

E-mail address: betting@fmb.unesp.br (L.E. Betting).
}

yses may be performed directly in the diffusion maps using an analogous methodology of the voxel-based morphometry (VBM) (Ashburner and Friston, 2000). The main drawback with this approach comes from incorrect registration of the images which, is considered a critical problem in DTI (Bookstein, 2001; Abe et al., 2010).

Another widely used technique is tract-based spatial statistics (TBSS) (Smith et al., 2006). This methodology consists in projecting the diffusion data onto a simplified map of the white-matter. The main objective of TBSS creation was to overcome the registration problem. However, limitations, especially concerning this issue and the direction of the tracts, still exist (Bach et al., 2014; Schwarz et al., 2014).

Improved voxel-based analyses of diffusion images may be achieved using tensor-based registration (Keihaninejad et al., 2013). Because whole brain voxel-wise analysis and TBSS are performed with different approaches, the main hypothesis is that they may show different aspects of the abnormalities in patients with 
MTLE. In addition, differences may also be observed depending on the diffusion parameter used. The objective of this investigation was a multimodal investigation of tensor-based registered DTI images of patients with MTLE.

\section{Methods}

This study was approved by the local ethics committee. Patients with refractory MTLE ( $n=24,16$ women, mean age $42 \pm 12$ years, range 20-68) and with hippocampal sclerosis (HS) were selected. Diagnosis of MTLE was performed according to clinical and electroencephalographic features following previous recommendations (Commission on Classification and Terminology of the International League Against Epilepsy, 1989; Berg et al., 2010). All patients had focal seizures which were detailed by clinical history obtained from patients or relatives. Patients with dual pathology or extra-temporal epilepsy were excluded. Control subjects $(n=36,18$ women, mean age $33 \pm 11$ years, range 21-57) without histories of neurologic disease were recruited from the local community. All subjects gave informed consent.

\subsection{Electroencephalogram (EEG)}

Interictal EEG was performed with a 32-channel recorder (Nihon Kohden, Tokyo, Japan). Electrodes were positioned according to the 10-20 international system of electrode placement and with additional Silverman's anterior temporal electrodes. All records were performed with $20 \mathrm{~min}$ long, with photic stimulation and hyperventilation.

\subsection{Magnetic resonance imaging (MRI)}

MRI acquisitions were performed using a $3 \mathrm{~T}$ scanner (Siemens, Verio, Erlangen, Germany) with a 12-channel head array coil.

HS was diagnosed by conventional visual examination based on two main sequences: coronal perpendicular to the long axis of the hippocampus defined at the sagittal image Short T1 inversion recovery (STIR; slice thickness, $2.2 \mathrm{~mm}$; field-of-view (FOV), $180 \mathrm{~mm}$; matrix size, $230 \times 256$; repetition time (TR), $2100 \mathrm{~ms}$; echo time (TE), $9.5 \mathrm{~ms}$; inversion time (TI), $499 \mathrm{~ms}$; flip angle, $150^{\circ}$; acquisition time, $3.36 \mathrm{~min}$ ) and $\mathrm{T} 2$ periodically rotated overlapping parallels lines with enhanced reconstruction (BLADE; slice thickness, $2.2 \mathrm{~mm}$; FOV, $180 \mathrm{~mm}$; matrix size, $230 \times 256$; TR, $4000 \mathrm{~ms}$; $\mathrm{TE}, 135 \mathrm{~ms}$; flip angle, $120^{\circ}$; acquisition time, $3.22 \mathrm{~min}$ ).

For hippocampal and whole brain volumetry, threedimensional Magnetization Prepared Rapid Gradient Echo T1 sequences (MPRAGE; 192 sagittal slices; slice thickness, $1 \mathrm{~mm}$; in plane resolution, $0.5 \times 0.5 \mathrm{~mm}$; FOV, $256 \mathrm{~mm}$; matrix size, $256 \times 256$; TR, $2300 \mathrm{~ms}$; TE, $2.47 \mathrm{~ms}$; TI, $1100 \mathrm{~ms}$; flip angle, 9॰ acquisition time, $5.21 \mathrm{~min}$ per volume) were used.

Diffusion weighted images were acquired using single-shot echo planar imaging (65 axial slices; slice thickness, $2 \mathrm{~mm}$; in plane resolution, $1.8 \times 1.8 \mathrm{~mm}$; 12 non collinear diffusion gradients direction, b-value $1000 \mathrm{~s} / \mathrm{mm}^{2}$; FOV, $230 \mathrm{~mm}$; matrix size $128 \times 128 \mathrm{~mm}$; TR, $9100 \mathrm{~ms}$; TE, $96 \mathrm{~ms}$; TI, $200 \mathrm{~ms}$; flip angle, 150 ; 4 averages to improve signal-to-noise ratio; total acquisition time, $8.21 \mathrm{~min}$ ). Images were acquired in Digital Imaging and Communications in Medicine (DICOM) format and transformed into ANALYZE and the Neuroimaging Informatics Technology Initiative (NIfTI) format using MRIcron software (Rorden and Brett, 2000).

\subsection{Hippocampal volumetry}

The FreeSurfer (http://surfer.nmr.mgh.harvard.edu/, version 5.3, developed at the Martinos Center for Biomedical Imaging by the Laboratory for Computational Neuroimaging, Charlestown,
MA, U.S.A.) image analysis suite was used to obtain hippocampal volumes and total intracranial volumes (TIV). Volumetry was performed to confirm HS and to group the patients according to the most atrophic hippocampus for bilateral HS. The standard processing pipeline implemented in the software was used. ANALYZE images were imported into the software, and they automatically underwent several processing steps including motion correction, non-brain tissue removal, automatic registration into Talairach space, segmentation of subcortical structures including the hippocampus, and tessellation of gray matter white matter boundaries (Fischl et al., 2002; Fischl et al., 2004).

Hippocampal volumes were normalized according to the TIV. An asymmetry index was calculated by: $2 \mathrm{x}$ (right hippocampus left hippocampus $) \div$ (right hippocampus + left hippocampus). The volumes and the asymmetry index obtained for each individual were standardized according to the value of normal controls using a z-score transformation.

\subsection{Diffusion tensor imaging processing}

FSL software (FMRIB Software Library, www.fmrib.ox.ac.uk/fsl, version 5.0, created by the Analysis Group, FMRIB, Oxford, U.K.) was used to obtain the main diffusion maps (Jenkinson et al., 2012). NifTI images were imported and submitted to brain extraction followed by eddy currents correction using the Brain Extraction Tool (BET) and FMRIB's Diffusion Toolbox (FDT) (Smith, 2002; Behrens et al., 2003). Finally, a diffusion tensor model was fitted at each voxel using DTIFIT (Behrens et al., 2003). After these steps, the DTI parameters obtained were imported into DTI-TK software (www. nitrc.org/projects/dtitk, developed at Penn Image Computing and Science Laboratory, University of Pennsylvania, Philadelphia, PA, U.S.A.) for further processing. This software provides an algorithm to register DTI images aligning white matter tracts by combining the fiber orientation in each voxel (Keihaninejad et al., 2013; Wang et al., 2011).

Using DTI-TK, a customized template was created. All images were submitted to an initial rigid alignment with a template based on 550 normal individuals with ages between 20 and 80 years (Zhang et al., 2010). To obtain the customized template, images were subsequently submitted to affine linear alignment and to a diffeomorphic registration algorithm with 6 iterations. After the creation of this specific template, it was individually submitted to rigid, affine and diffeomorphic alignment with a specific enhanced DTI template in ICBM-152 space (International Consortium for Brain Mapping, Montreal Neurological Institute, MNI) (Zhang et al., 2011). Finally, deformation fields obtained for the creation and ICBM-152 transformation of the template were applied for each individual in the study. The final results were aligned images in the standard space with $1 \mathrm{~mm}$ isotropic voxels. For each subject, diffusion parameter maps of fractional anisotropy (FA), axial diffusivity $(A D)$, radial diffusivity $(R D)$ and mean diffusivity (MD) were finally extracted and used for statistical analysis.

\subsection{Statistical analysis}

Two types of voxel-based comparisons were conducted. A whole brain voxel-based analysis was performed using SPM12 (www.fil. ion.ucl.ac.uk/spm, Wellcome Trust Centre for Neuroimaging, University College London, London, U.K.) running under the Matlab R2012b platform (MathWorks, Natick, MA, U.S.A.). In this method, diffusion images were smoothed with an $8 \mathrm{~mm}$ Gaussian filter (Full Width at Half Maximum). For each diffusion parameter, comparisons between patients and controls were performed using a full factorial design with two contrasts (searching for areas of increased and decreased abnormalities). 
Table 1

Clinical, electroencephalographic and neuroimaging features of 24 patients with mesial temporal lobe epilepsy.

\begin{tabular}{|c|c|c|c|c|c|c|c|c|c|c|c|c|}
\hline $\mathrm{N}$ & HS & First & Freq & Medications & EEG & Age & Gender & RH vol & $\mathrm{RH} z$ & LH vol & $\mathrm{LH} \mathrm{z}$ & $\mathrm{AI} \mathrm{z}$ \\
\hline 1 & $\mathrm{~L}$ & 8 & 0 & CBZ 1200, LTG 100, CLB 60 & Normal & 52 & Women & 3766 & 0.8 & 2422 & -4.3 & 9.1 \\
\hline 2 & $\mathrm{~L}$ & 25 & 3 & LTG 300, CLB 20 & Left & 58 & Men & 3894 & -0.3 & 2866 & -3.8 & 6.2 \\
\hline 3 & $\mathrm{R}$ & 1 & 2 & TPM 300, PHT 300 & Right, Slow & 43 & Women & 2911 & -3.2 & 4213 & 1.6 & -8.6 \\
\hline 4 & $\mathrm{R}$ & 11 & 2 & OXC 2100, VPA 1000, CLB 20 & Bilateral & 33 & Men & 2809 & -4.1 & 3700 & -1.1 & -6.5 \\
\hline 5 & $\mathrm{~L}$ & 12 & 2 & LTG 450, CLB 40 & Left & 28 & Women & 3919 & -0.4 & 2532 & -5.2 & 9.0 \\
\hline 6 & $\mathrm{~L}$ & 10 & 4 & CLB 20, PB 50, CBZ 1000 & Left & 37 & Women & 4469 & 1.4 & 3907 & -0.1 & 2.5 \\
\hline 7 & $\mathrm{~L}$ & 7 & 3 & CBZ 1000 & Slow & 22 & Women & 4122 & 1.2 & 2805 & -3.5 & 7.9 \\
\hline 8 & $\mathrm{~L}$ & 3 & 3 & PHT 300, CLB 10 & Left & 58 & Men & 4153 & 3.0 & 2749 & -2.4 & 8.5 \\
\hline 9 & $\mathrm{~B}$ & 2 & 0 & CBZ 1200, CLB 10 & Right & 49 & Women & 2489 & -4.4 & 2411 & -5.0 & 0.2 \\
\hline 10 & $\mathrm{R}$ & 17 & 8 & CBZ 600, CLB 20 & Right & 26 & Men & 3846 & -2.6 & 4248 & -1.4 & -2.7 \\
\hline 11 & $\mathrm{R}$ & 10 & 4 & CBZ 1200 & Right & 39 & Women & 3814 & -2.0 & 4633 & 0.8 & -4.8 \\
\hline 12 & $\mathrm{R}$ & 13 & 6 & CBZ 600, LTG 400 & Right & 61 & Women & 3629 & -1.3 & 4600 & 2.5 & -5.7 \\
\hline 13 & $\mathrm{R}$ & 28 & 20 & CBZ 1200, CLB 20 & Right & 42 & Women & 4133 & -0.8 & 4405 & 0.3 & -2.0 \\
\hline 14 & $\mathrm{~L}$ & 10 & 4 & CBZ 1200, CLB 40 & Left & 39 & Women & 4392 & 0.7 & 2884 & -4.2 & 8.6 \\
\hline 15 & $\mathrm{~L}$ & 10 & 2 & PB 100, CBZ 400 & Left & 41 & Men & 4304 & 0.9 & 3349 & -2.2 & 5.0 \\
\hline 16 & $\mathrm{R}$ & 12 & 20 & CBZ 400, CLB 40, TPM 400 & Right & 37 & Women & 3110 & -3.9 & 3967 & -1.2 & -5.8 \\
\hline 17 & $\mathrm{~L}$ & 5 & 12 & CBZ 1200, CLB 20 & Left & 37 & Women & 3353 & -1.0 & 2372 & -4.9 & 7.1 \\
\hline 18 & $\mathrm{R}$ & 21 & 10 & CBZ 200, CLB 60 & Bilateral & 40 & Women & 3017 & -3.5 & 4494 & 1.7 & -9.2 \\
\hline 19 & B & 7 & 1 & TPM 125, CLB 10 & Bilateral & 60 & Women & 2918 & -3.9 & 2205 & -6.7 & 5.6 \\
\hline 20 & B & 5 & 4 & CBZ 1200 & Left & 43 & Women & 2442 & -4.9 & 2231 & -6.1 & 1.5 \\
\hline 21 & $\mathrm{~L}$ & 2 & 2 & CBZ 600, PHT 200, PB 100 & - & 43 & Men & 3899 & -0.6 & 2503 & -5.4 & 9.1 \\
\hline 22 & $\mathrm{R}$ & 42 & 8 & CBZ 1000 & Bilateral & 58 & Women & 3420 & -1.2 & 3852 & 0.6 & -3.1 \\
\hline 23 & $\mathrm{~L}$ & 7 & - & CBZ 1000 & Left & 68 & Men & 3818 & -1.5 & 2835 & -4.7 & 6.0 \\
\hline 24 & $\mathrm{R}$ & 4 & 3 & CBZ 1200, CLB 20 & Normal & 20 & Men & 2577 & -5.2 & 3888 & -1.1 & -9.4 \\
\hline
\end{tabular}

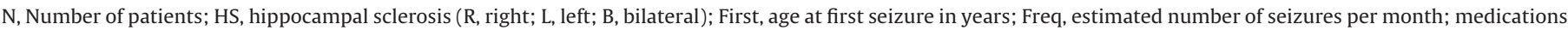

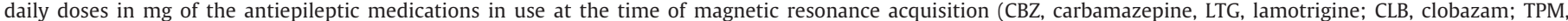

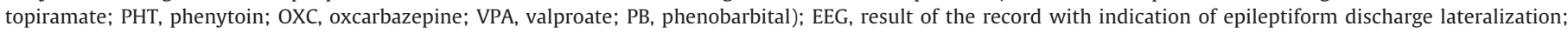

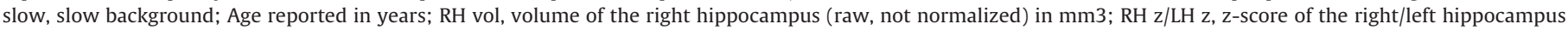
after normalization; LH vol, volume of the left hippocampus (raw, not normalized) in mm3; AI z, z-score of the asymmetry index after normalization.

The second type of voxel-based analysis used was TBSS. Based on the FA images, a mean skeleton of the white matter of the whole group was created. For each parameter, the values of white matter tracts of all individuals were projected in this FA skeleton. Comparisons between each parameter of patients and controls were performed using Randomise, an FSL tool for nonparametric permutation inference (Winkler et al., 2014). Two contrasts searching for areas of increased and decreased abnormalities and 5000 permutations were used.

After the statistical analysis, a Talairach atlas was used to identify the nearest gray matter anatomical structure (Talairach and Tournoux, 1988). For the identification of the main fasciculi involved, the Johns Hopkins University (JHU) white-matter tractography atlas was used (Mori, 2005). This probabilistic atlas identified 20 main structures in 28 individuals (Mori, 2005).

Finally, to confirm the results a tract-specific analysis (TSA) was performed for the main tracts depicted in the previous steps. Using DTI-TK, samples of the white matter and tract-specific attributes across the whole population were obtained. The mean value was projected onto a medial two dimensional model representing the real tridimensional tracts. For each individual the values corresponding to the spokes from each vertex of the mesh were obtained and used for the analysis (Yushkevich et al., 2008). Statistical analysis was performed comparing the tracts of patients and controls using a general linear model with two contrasts (increased and decreased differences).

For the three types of analyses, age, sex and TIV were introduced as covariates. The level of significance selected was $\mathrm{p}<0.05$ corrected for multiple comparisons (Family-Wise Error and for TBSS with additional Threshold-Free Cluster Enhancement).

\section{Results}

\subsection{Clinical and EEG findings}

The mean age of recurrent seizures onset was $10 \pm 9$ years (range, 1-42). At the time of MRI acquisition, the mean fre- quency of focal seizures estimated according to clinical history was $5 \pm 5$ per month (0-20) and the patients were using a mean of $2 \pm 1$ antiepileptic drugs (1-3). Interictal EEG showed epileptiform discharges in the left anterior temporal region in 9 patients. Meanwhile, 7 patients showed discharges in the right anterior temporal region, and 4 had bilateral discharges. Two patients presented with normal EEGs, 1 patient had abnormal background activity and 1 patient was not subjected to the exam. The clinical and EEG findings are reported in Table 1.

\subsection{MRI findings}

Hippocampal volumetry confirmed that 10 patients presented with left HS, 11 with right HS, and 3 with bilateral HS with left predominance. The mean volume of the left hippocampus was $4315 \pm 363(3428-4961)$ for the controls and $3336 \pm 848$ (2205-4633) $\mathrm{mm}^{3}$ for the patients. The mean volume of the right hippocampus was $4412 \pm 362(3682-5268)$ for the controls and $3550 \pm 626(2442-4469) \mathrm{mm}^{3}$ for the patients. After normalization, the $\mathrm{z}$-scores obtained for the patients were $-2.1 \pm 2.7$ ( $-6.7-2.8)$ for the left and $-1.4 \pm 2.2(-5.2-3)$ for the right hippocampus. The mean asymmetry index was $1.1 \pm 5.6(-9.4-9.1)$. These findings are detailed in Table 1 and Fig. 1.

\subsection{Whole brain voxel-wise analysis}

For the whole group of patients, we obtained: one cluster of increased $\mathrm{AD}$, volume of $290 \mathrm{~mm}^{3}$, main localization in the right parahippocampal gyrus $(\mathrm{x}=31, \mathrm{y}=-18, \mathrm{z}=-24$; $\mathrm{T}$ value $=6.11$; $\mathrm{Z}=5.32 ; \mathrm{p}=0.001$ ); two clusters of increased $\mathrm{RD}$, total volume of $1102 \mathrm{~mm}^{3}$, main localization in the right parahippocampal gyrus $(x=28, y=-16, z=-25 ; T$ value $=6.65 ; Z=5.67 ; p<0.0001)$; two clusters of increased MD, total volume of $782 \mathrm{~mm}^{3}$, main localization in the right parahippocampal gyrus $(x=29, y=-17$, $\mathrm{z}=-24 ; \mathrm{T}$ value $=6.52 ; \mathrm{Z}=5.59 ; \mathrm{p}<0.0001$ ); five clusters of reduced FA, total volume of $1685 \mathrm{~mm}^{3}$, main localization in the left temporal lobe involving the uncinate and inferior fronto-occipital fasci- 

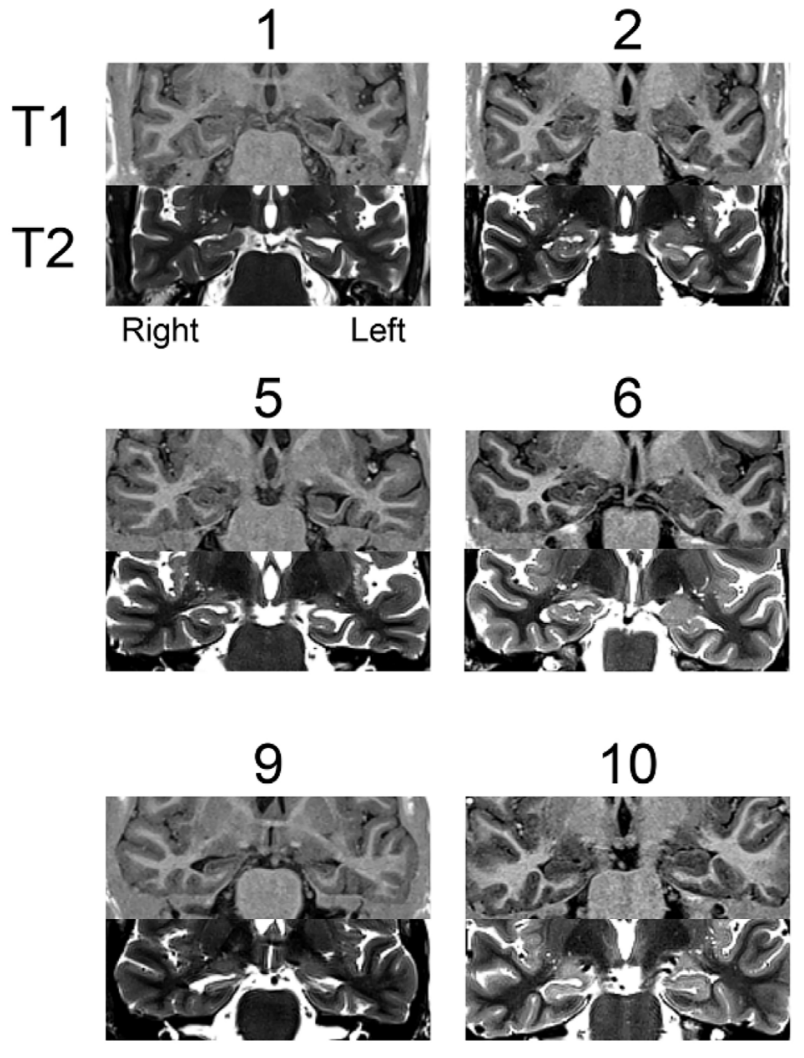

13

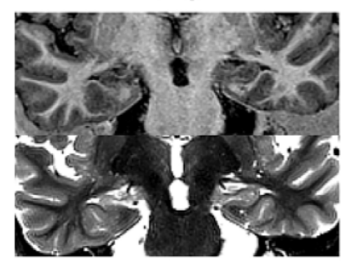

17
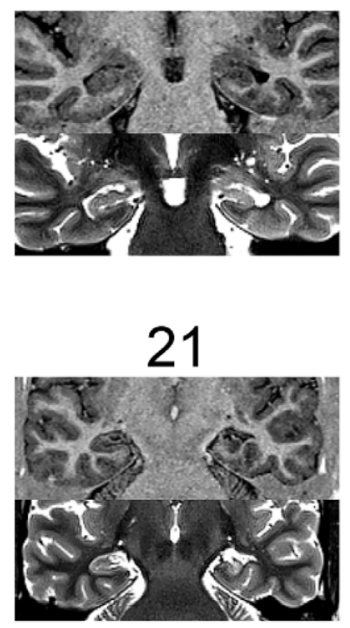
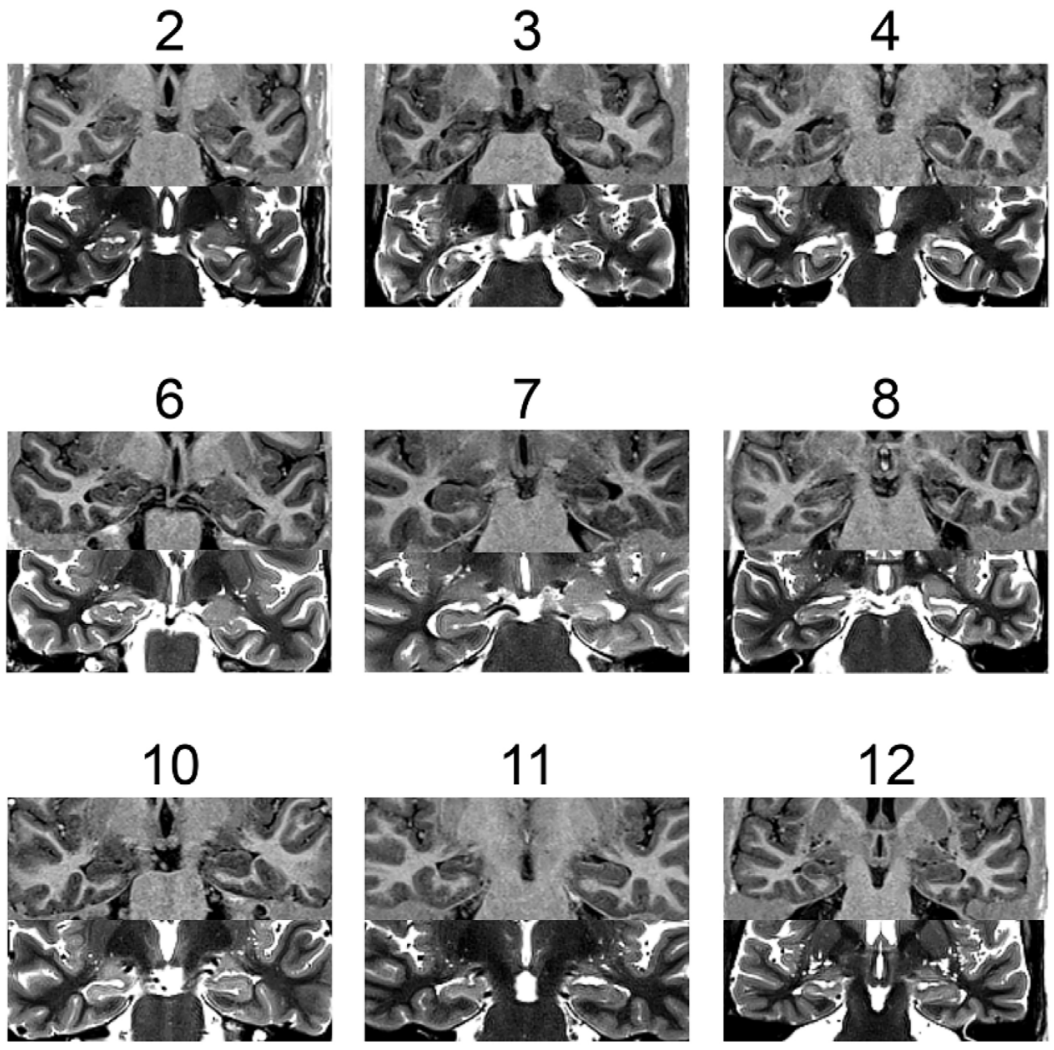

12

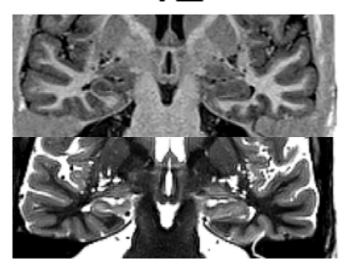

14

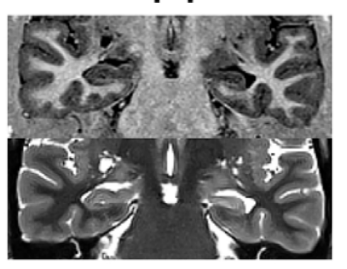

18
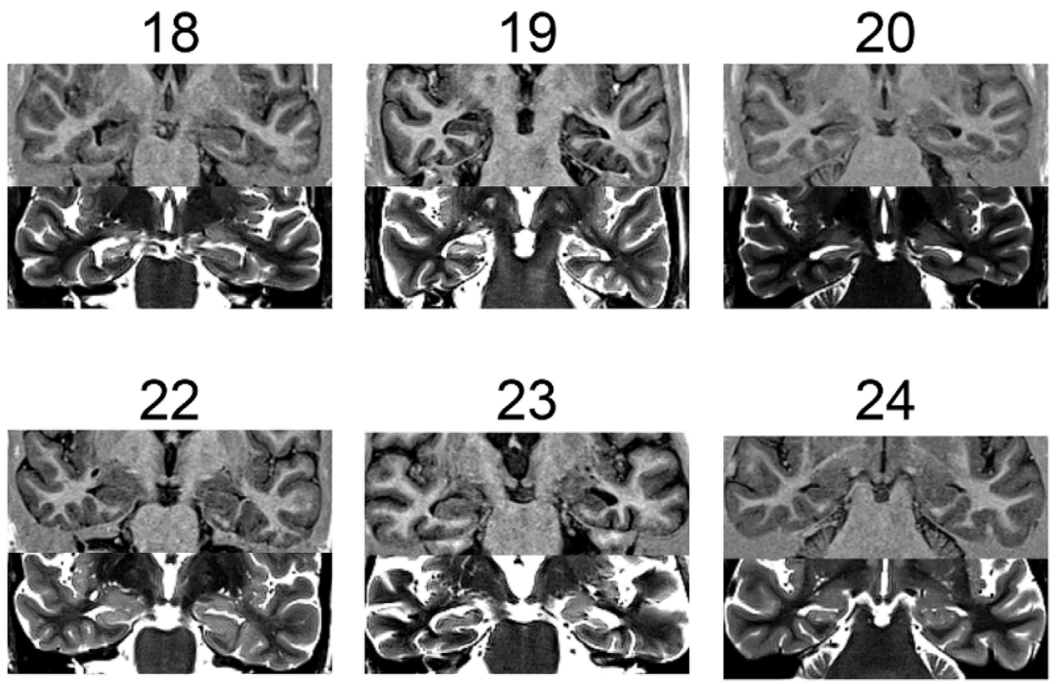

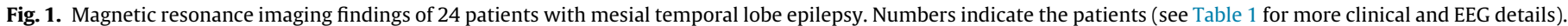

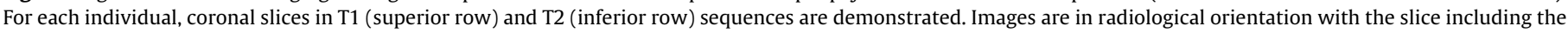
anterior portion of the hippocampus. 
Table 2

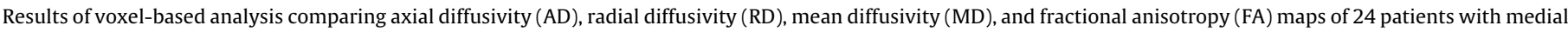
temporal lobe epilepsy to those of 36 controls according to two methodologies: whole brain voxel-wise analysis and tract-based spatial statistics.

\begin{tabular}{|c|c|c|c|c|c|c|}
\hline \multirow[t]{2}{*}{ Method } & \multirow[t]{2}{*}{ Parameter } & \multirow[t]{2}{*}{ Volume $\left(\mathrm{mm}^{3}\right)$} & \multirow[t]{2}{*}{ P-value } & \multirow[t]{2}{*}{ Coordinates } & \multicolumn{2}{|l|}{ Localization } \\
\hline & & & & & Talairach & JHU \\
\hline \multirow{10}{*}{$\begin{array}{l}\text { Whole brain } \\
\text { voxel-wise analysis }\end{array}$} & $\mathrm{AD}$ & 290 & 0.001 & $31,-18,-24$ & Parahippocampal & Right cingulum (hippocampus) \\
\hline & $\mathrm{RD}$ & 758 & $<0.0001$ & $28,-16,-25$ & Parahippocampal & Right cingulum (hippocampus) \\
\hline & & 344 & $<0.0001$ & $-34,-10,-15$ & Temporal lobe & $\begin{array}{l}\text { Left inferior fronto-occipital, uncinate, } \\
\text { inferior longitudinal }\end{array}$ \\
\hline & MD & 595 & $<0.0001$ & $29,-17,-24$ & Parahippocampal & Right cingulum (hippocampus) \\
\hline & & 187 & 0.003 & $-32,-10,-15$ & Parahippocampal & $\begin{array}{l}\text { Left inferior longitudinal, anterior } \\
\text { thalamic radiation }\end{array}$ \\
\hline & FA & 719 & $<0.0001$ & $-33,-11,-13$ & Sub-lobar & Left uncinate, inferior fronto-occipital \\
\hline & & 624 & $<0.0001$ & $34,-3,-15$ & Temporal lobe & $\begin{array}{l}\text { Right inferior fronto-occipital, anterior } \\
\text { thalamic radiation, inferior } \\
\text { longitudinal }\end{array}$ \\
\hline & & 234 & 0.001 & $-40,-33,-3$ & Temporal lobe & $\begin{array}{l}\text { Left inferior fronto-occipital, inferior } \\
\text { longitudinal, superior longitudinal }\end{array}$ \\
\hline & & 57 & 0.011 & $-22,-6,32$ & Sub-lobar & $\begin{array}{l}\text { Left inferior longitudinal, anterior } \\
\text { thalamic radiation }\end{array}$ \\
\hline & & 51 & 0.012 & $-26,-28,-2$ & Frontal lobe & Left superior longitudinal \\
\hline \multirow[t]{9}{*}{$\begin{array}{l}\text { Tract-based spatial } \\
\text { statistics }\end{array}$} & $\mathrm{AD}$ & 423 & 0.028 & $-39,7,-30$ & Superior temporal & $\begin{array}{l}\text { Left inferior longitudinal, uncinate, } \\
\text { inferior fronto-occipital }\end{array}$ \\
\hline & RD & 1132 & 0.001 & $-38,-10,-19$ & Temporal lobe & $\begin{array}{l}\text { Left anterior thalamic radiation, } \\
\text { inferior longitudinal, inferior } \\
\text { fronto-occipital }\end{array}$ \\
\hline & & 412 & 0.001 & $28,4,-35$ & Uncus & Right inferior longitudinal \\
\hline & MD & 254 & 0.008 & $28,4,-35$ & Uncus & Right inferior longitudinal \\
\hline & & 69 & 0.009 & $-42,-7,-22$ & Temporal lobe & $\begin{array}{l}\text { Left inferior longitudinal, uncinate, } \\
\text { superior longitudinal, inferior } \\
\text { fronto-occipital }\end{array}$ \\
\hline & FA & 17581 & $<0.001$ & $-39,-5,-36$ & Temporal lobe & Left inferior longitudinal \\
\hline & & 1332 & $<0.001$ & $42,-3,-35$ & Middle temporal & Right inferior longitudinal \\
\hline & & 523 & 0.001 & $39,-40,18$ & Superior temporal & Right superior longitudinal \\
\hline & & 205 & 0.001 & $32,-49,18$ & Temporal lobe & $\begin{array}{l}\text { Right inferior fronto-occipital, inferior } \\
\text { longitudinal, superior longitudinal, } \\
\text { forceps major }\end{array}$ \\
\hline
\end{tabular}

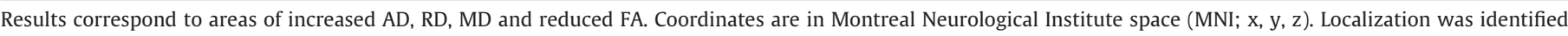
according to the Talairach atlas and the Johns Hopkins University white-matter tractography atlas (Talairach and Tournoux, 1988; Mori, 2005).
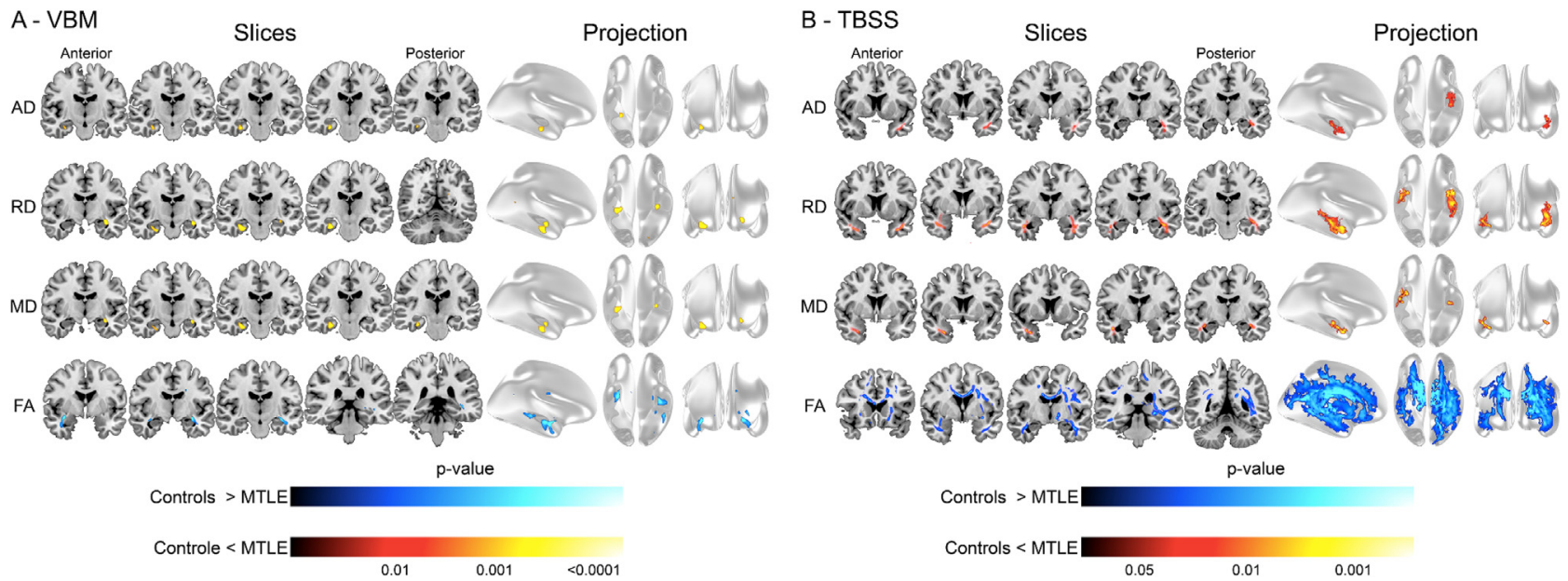

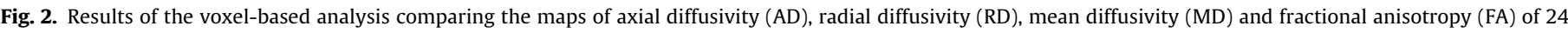

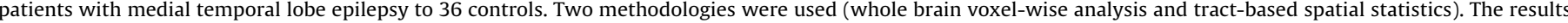

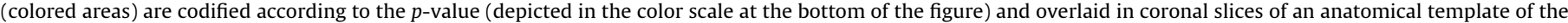
brain (Slices). A tridimensional inflated brain model also shows the projection of all abnormal areas. Orientation is radiological.

culi $(x=-33, y=-11, z=-13 ; \mathrm{T}$ value $=6.44 ; \mathrm{Z}=5.54 ; \mathrm{p}<0.0001)$. Table 2 and Fig. 2 show these findings. Areas of reduced AD, RD, MD and increased FA were not disclosed.

For patients with right MTLE, we obtained: two clusters of increased $\mathrm{AD}$, volume of $203 \mathrm{~mm}^{3}$, main localization in the right hippocampus and parahippocampal gyrus $(x=30, y=-17$, $z=-24 ;$ T value $=5.9 ; Z=5.16 ; p=0.003)$; two clusters of increased $\mathrm{RD}$, total volume of $360 \mathrm{~mm}^{3}$, main localization in the right hip- pocampus and parahippocampal gyrus $(x=26, y=-17, z=-25 ; T$ value $=6.36 ; Z=5.47 ; p=0.001)$; three clusters of increased $M D$, total volume of $338 \mathrm{~mm}^{3}$, main localization in the right hippocampus and parahippocampal gyrus $(x=27, y=-17, z=-25$; $T$ value $=6.30 ; Z=5.43 ; p=0.001)$; two clusters of reduced $F A$, total volume of $371 \mathrm{~mm}^{3}$, main localization in the right temporal lobe involving the uncinate, inferior fronto-occipital and inferior longitudinal fasciculi $(\mathrm{x}=-33, \mathrm{y}=-1, \mathrm{z}=-13 ; \mathrm{T}$ value $=6.81 ; \mathrm{Z}=5.76 ; \mathrm{p}$ 


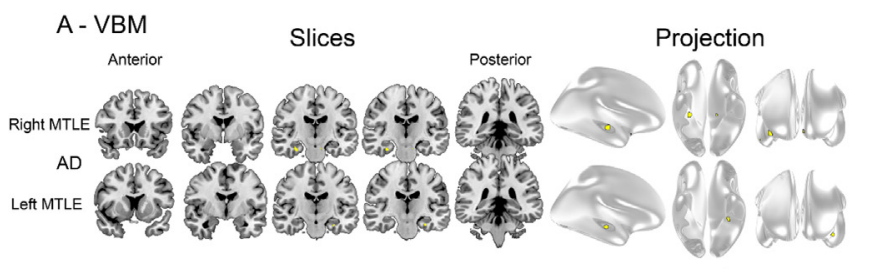

B - TBSS
Anterior

AD (no statistically significant areas)
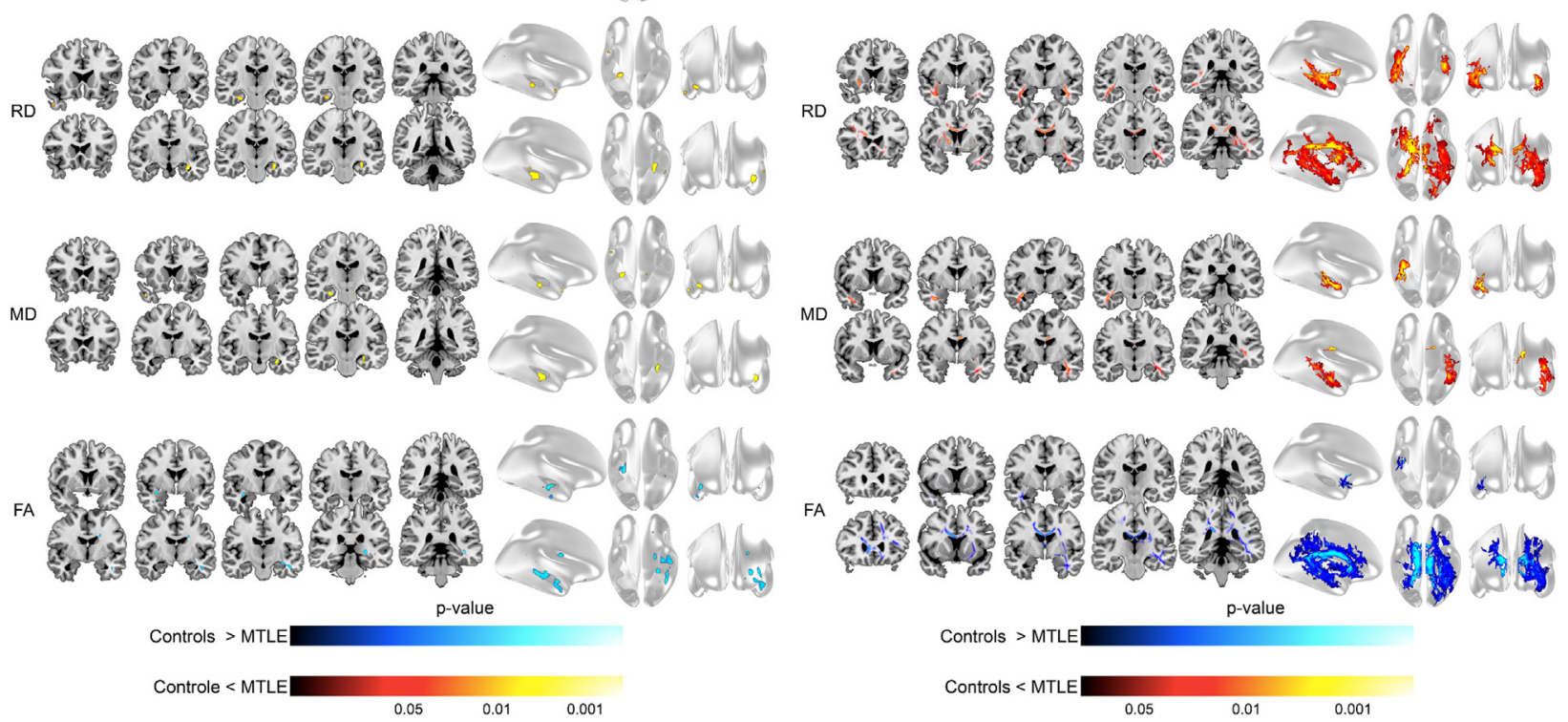

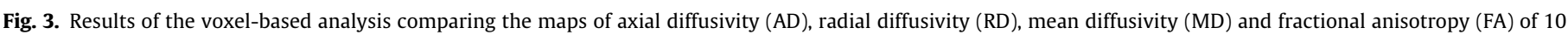

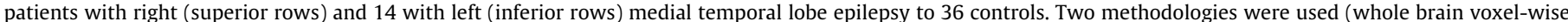

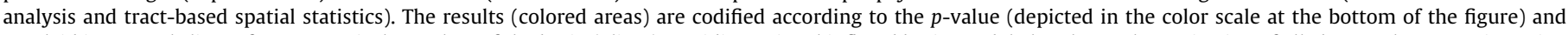

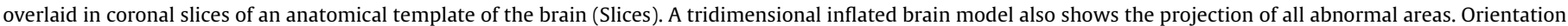
is radiological.

$<0.0001)$. Fig. 3 show these findings. Areas of reduced AD, RD, MD and increased FA were not disclosed.

For patients with left MTLE, we obtained: one cluster of increased $\mathrm{AD}$, volume of $81 \mathrm{~mm}^{3}$, main localization in the left hippocampus and parahippocampal gyrus $(x=-29, y=-17, z=-23 ; T$ value $=5.66 ; Z=5 ; p=0.009$ ); two clusters of increased $R D$, total volume of $735 \mathrm{~mm}^{3}$, main localization in the left parahippocampal gyrus involving the anterior thalamic radiation $(x=-32, y=-12$, $\mathrm{z}=-18 ;$ T value $=6.50 ; \mathrm{Z}=5.56 ; \mathrm{p}<0.0001)$; one cluster of increased $\mathrm{MD}$, total volume of $456 \mathrm{~mm}^{3}$, main localization in the left parahippocampal gyrus involving the inferior longitudinal fasciculus $(x=-30, y=-12, z=-18 ; T$ value $=6.17 ; Z=5.34 ; p=0.005)$; four clusters of reduced FA, total volume of $1505 \mathrm{~mm}^{3}$, main localization in the left parahippocampal gyrus involving the inferior longitudinal fasciculi $(x=-27, y=-28, z=0 ;$ T value $=5.82 ; Z=5.11$; $\mathrm{p}=0.001$ ). Fig. 3 show these findings. Areas of reduced AD, RD, MD and increased FA were not disclosed.

\subsection{TBSS analysis}

For the whole group of patients, it was obtained: one cluster of increased $\mathrm{AD}$, volume of $423 \mathrm{~mm}^{3}$, localization in the left superior temporal gyrus involving the inferior longitudinal, uncinate and inferior fronto-occipital fasciculi $(x=-39, y=7, z=-30 ; p=0.028)$; two clusters of increased RD, total volume of $1544 \mathrm{~mm}^{3}$, main localization in the left temporal lobe involving the anterior thalamic radiation, inferior longitudinal and inferior fronto-occipital fasciculi $(x=-38, y=-10, z=-19 ; p=0.001)$; two clusters of increased $\mathrm{MD}$, total volume of $323 \mathrm{~mm}^{3}$, main localization in the right uncus involving the inferior longitudinal fasciculus $(x=28, y=4, z=-35$; $\mathrm{p}=0.008$ ); four clusters of reduced $\mathrm{FA}$, total volume of $19641 \mathrm{~mm}^{3}$, main localization in the left temporal lobe involving the inferior longitudinal fasciculus $(\mathrm{x}=-39, \mathrm{y}=-5, \mathrm{z}=-36 ; \mathrm{p}<0.001)$. Table 2 and Fig. 2 show these findings. Areas of reduced AD, RD, MD and increased FA were not disclosed.

For patients with right MTLE, it was obtained: two clusters of increased RD, volume of $2663 \mathrm{~mm}^{3}$, main localization in the right temporal lobe involving the inferior longitudinal fasciculus $(x=42$, $y=-3, z=-27 ; p=0.008)$; one cluster of increased MD, total volume of $675 \mathrm{~mm}^{3}$, main localization in the right temporal lobe involving the inferior longitudinal fasciculus $(x=31, y=8, z=-34$; $\mathrm{p}=0.023$ ); one cluster of reduced $\mathrm{FA}$, total volume of $514 \mathrm{~mm}^{3}$, main localization in the right middle temporal gyrus involving the inferior longitudinal fasciculus $(x=42, y=-3, z=-35 ; p=0.005)$. Fig. 3 shows these findings. Areas of reduced AD, RD, MD and increased $\mathrm{AD}$ and $\mathrm{FA}$ were not disclosed.

For patients with left MTLE, it was obtained: four clusters of increased $\mathrm{RD}$, volume of $8478 \mathrm{~mm}^{3}$, main localization in the left superior temporal lobe involving the inferior longitudinal fasciculus and anterior thalamic radiation $(x=-34, y=4$, $\mathrm{z}=-31 ; \mathrm{p}=0.001$ ); three clusters of increased MD, total volume of $1643 \mathrm{~mm}^{3}$, main localization in the left temporal lobe involving mainly the inferior longitudinal fasciculus $(x=-42, y=-7, z=-22$; $\mathrm{p}=0.011$ ); one cluster of reduced $\mathrm{FA}$, total volume of $17928 \mathrm{~mm}^{3}$, main localization in the left temporal lobe involving the inferior longitudinal fasciculus $(x=-39, y=-5, z=-36 ; p<0.001)$. Fig. 3 shows these findings. Areas of reduced AD, RD, MD and increased $\mathrm{AD}$ and FA were not disclosed.

\subsection{TSA analysis}

TSA showed increased AD, RD and MD as well as reduced FA in patients with MTLE. For the whole group analysis, RD $\left(5442 \mathrm{~mm}^{2}\right)$ and $\mathrm{MD}\left(5496 \mathrm{~mm}^{2}\right)$ were the parameters with the larger affected 
Table 3

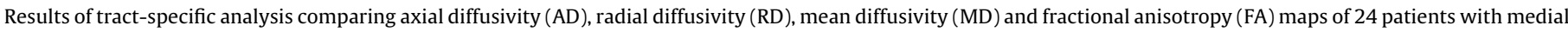
temporal lobe epilepsy to those of 36 controls.

\begin{tabular}{|c|c|c|c|c|c|}
\hline Parameter & Area $\left(\mathrm{mm}^{2}\right)$ & P-value & T value (mean) & Hemisphere & Localization \\
\hline \multirow[t]{3}{*}{$\mathrm{AD}$} & 645 & 0.002 & 2.49 & left & Inferior fronto-occipital \\
\hline & 316 & 0.002 & 2.88 & left & Uncinate \\
\hline & 319 & 0.0012 & 2.58 & right & Uncinate \\
\hline \multirow[t]{10}{*}{$\mathrm{RD}$} & 217 & 0.044 & 1.87 & left & Inferior longitudinal \\
\hline & 813 & 0.003 & 2.17 & right & Inferior longitudinal \\
\hline & 425 & 0.02 & 1.98 & right & Inferior longitudinal \\
\hline & 1108 & 0.001 & 2.87 & left & Inferior fronto-occipital \\
\hline & 637 & 0.008 & 1.87 & left & Inferior fronto-occipital \\
\hline & 797 & 0.007 & 2.98 & right & Inferior fronto-occipital \\
\hline & 419 & 0.007 & 2.08 & left & Superior longitudinal \\
\hline & 300 & 0.024 & 1.81 & left & Superior longitudinal \\
\hline & 301 & 0.005 & 2.45 & left & Uncinate \\
\hline & 425 & $<0.0001$ & 3.03 & right & Uncinate \\
\hline \multirow[t]{8}{*}{ MD } & 949 & 0.005 & 2.18 & right & Inferior longitudinal \\
\hline & 369 & 0.043 & 2.03 & right & Inferior longitudinal \\
\hline & 1006 & 0.002 & 2.96 & left & Inferior fronto-occipital \\
\hline & 781 & 0.004 & 2.1 & left & Inferior fronto-occipital \\
\hline & 1132 & 0.007 & 2.89 & right & Inferior fronto-occipital \\
\hline & 496 & 0.003 & 1.89 & left & Superior longitudinal \\
\hline & 322 & 0.003 & 2.67 & left & Uncinate \\
\hline & 441 & $<0.0001$ & 2.98 & right & Uncinate \\
\hline \multirow[t]{5}{*}{ FA } & 291 & 0.032 & 1.83 & right & Inferior longitudinal \\
\hline & 864 & 0.0006 & 2.41 & left & Inferior fronto-occipital \\
\hline & 244 & 0.04 & 2.54 & right & Inferior fronto-occipital \\
\hline & 259 & 0.022 & 2.13 & left & Superior longitudinal \\
\hline & 180 & 0.017 & 2.77 & right & Uncinate \\
\hline
\end{tabular}

Uncinate

Superior Longitudinal

AD

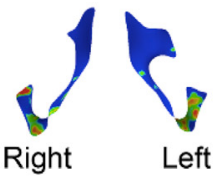

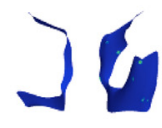
Right Left

$\mathrm{RD}$<smiles>CCCCCCC</smiles>

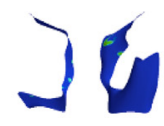

\section{Inferior}

Inferior

Right Lateral
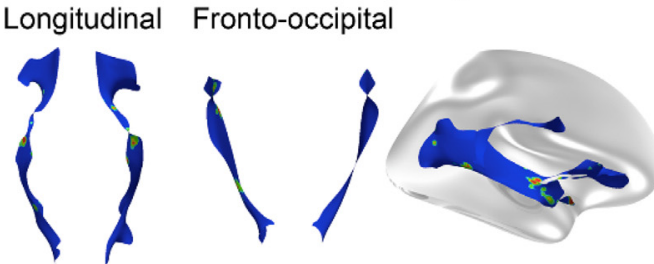

Inferior Axial

\section{Left Lateral}
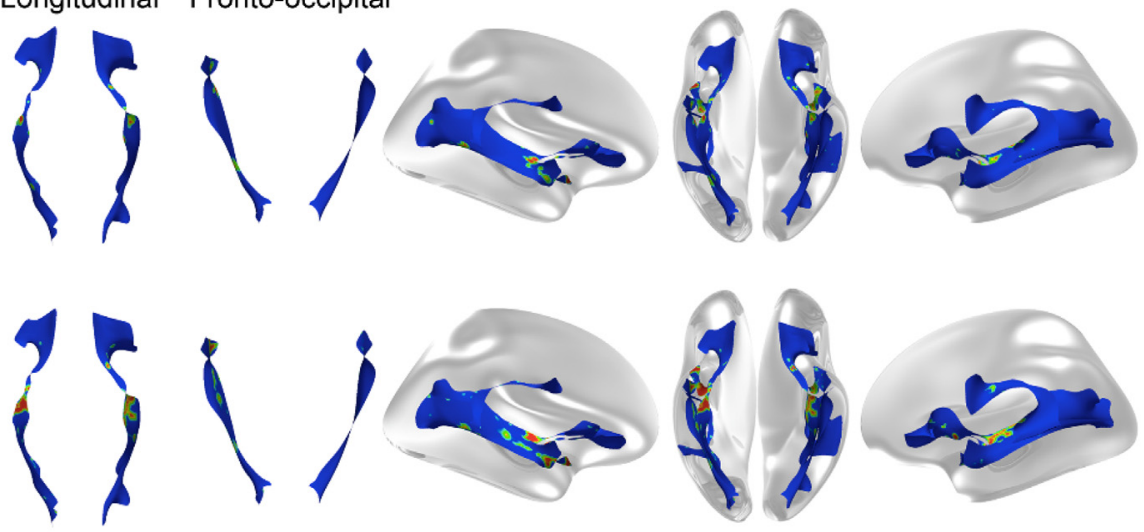

MD<smiles>C=CCCCC</smiles><smiles>[Li]</smiles>
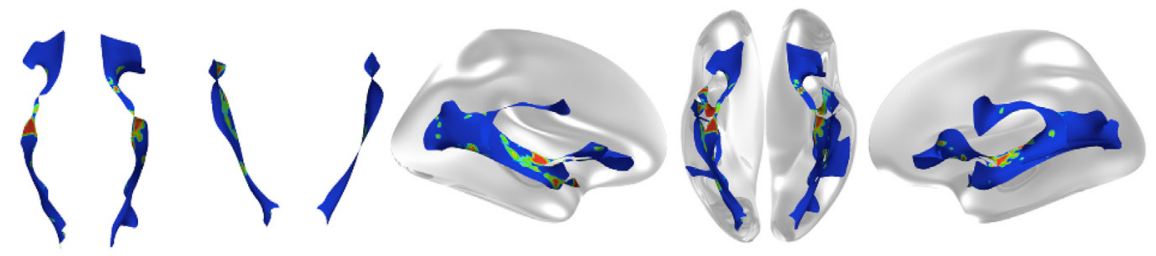

FA
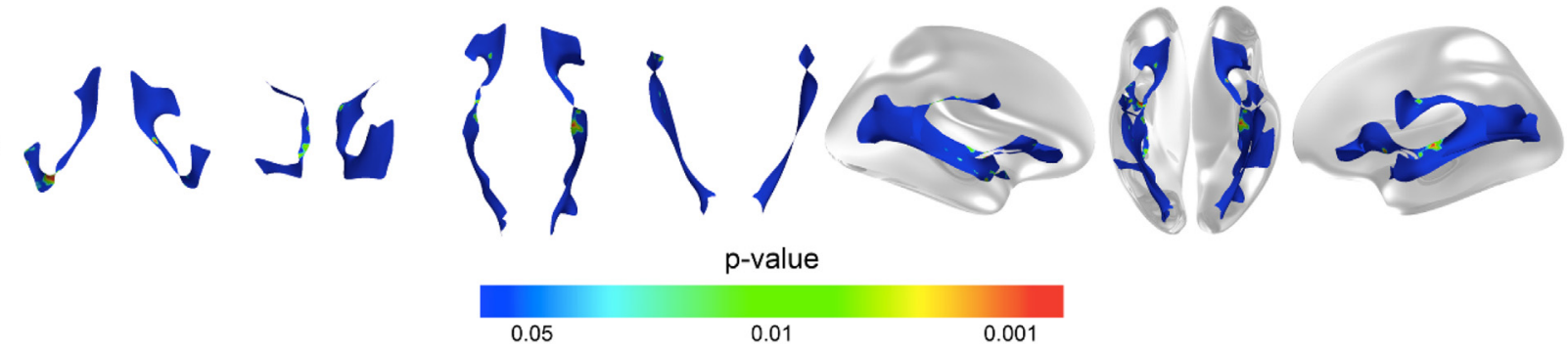

$p$-value

0.01

0.001

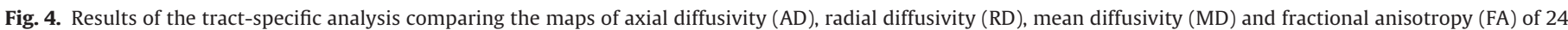

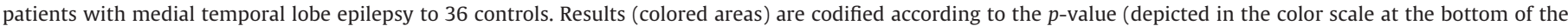
figure) and overlaid in the flattened tridimensional model of the fasciculi. An overlay of all fasciculi with a tridimensional inflated brain model is also depicted. 
areas, followed by FA $\left(1838 \mathrm{~mm}^{2}\right)$ and $\mathrm{AD}\left(1280 \mathrm{~mm}^{2}\right)$. The distribution of the abnormalities was mainly in inferior fronto-occipital $\left(7214 \mathrm{~mm}^{2}\right)$ and inferior longitudinal $\left(3064 \mathrm{~mm}^{2}\right)$ regions followed by the uncinate $\left(2304 \mathrm{~mm}^{2}\right)$ and superior longitudinal fasciculi $\left(1474 \mathrm{~mm}^{2}\right)$. There were no areas of reduced AD, RD or MD, nor of increased FA. Table 3 and Fig. 4 show these findings in detail.

For patients with right MTLE, RD $\left(3167 \mathrm{~mm}^{2}\right)$ and MD $\left(2812 \mathrm{~mm}^{2}\right)$ were the parameters with the larger affected areas, followed by FA $\left(1878 \mathrm{~mm}^{2}\right)$ and $\mathrm{AD}\left(272 \mathrm{~mm}^{2}\right)$. RD, MD and FA showed bilateral areas of abnormalities. The distribution of the abnormalities was mainly in inferior fronto-occipital $\left(3045 \mathrm{~mm}^{2}\right)$ and inferior longitudinal $\left(3010 \mathrm{~mm}^{2}\right)$ followed by the uncinate fasciculi $\left(2347 \mathrm{~mm}^{2}\right.$ ). Inferior fronto-occipital and inferior longitudinal fasciculi showed bilateral areas of abnormalities. There were no areas of reduced $A D, R D$ or MD, nor of increased FA.

For patients with left MTLE, RD $\left(4527 \mathrm{~mm}^{2}\right)$ and MD $\left(3578 \mathrm{~mm}^{2}\right)$ were the parameters with the larger affected areas, followed by AD $\left(1356 \mathrm{~mm}^{2}\right)$ and FA $\left(1298 \mathrm{~mm}^{2}\right)$. AD, RD and MD showed bilateral areas of abnormalities. The distribution of the abnormalities was mainly in inferior fronto-occipital $\left(4982 \mathrm{~mm}^{2}\right)$ and superior longitudinal $\left(2032 \mathrm{~mm}^{2}\right)$ followed by the uncinate $\left(1883 \mathrm{~mm}^{2}\right)$ and inferior longitudinal fasciculi $\left(1862 \mathrm{~mm}^{2}\right)$. Inferior fronto-occipital, inferior longitudinal and uncinate fasciculi showed bilateral areas of abnormalities. There were no areas of reduced $A D, R D$ or $M D$, nor of increased FA.

\section{Discussion}

In this investigation different patterns of diffusion abnormalities were observed in patients with MTLE depending on the methodology and the diffusion parameter used. For the voxel-based analysis investigation, FA and RD were the parameters that showed more abnormalities. Therefore, they should be routinely used in the quantitative investigation of diffusion abnormalities in MTLE.

Clinical interpretation of DTI parameters is complex and should be performed with care (Alexander et al., 2007). To maximize the specificity of the DTI investigation, the use of several parameters, such as FA, AD, and RD, is recommended. FA is reduced in several conditions and is considered a marker of fiber integrity but this is an over-simplification. FA basically indicates that orientation dependent aspects of the brain microstructure are different (Jones et al., 2013). Decreasing axonal density, increasing axonal caliber and reducing the degree of myelination should all lead to increased $\mathrm{RD}$ and reduced FA (Jones et al., 2013). AD indicates the main fiber orientation. Most investigations of patients with MTLE have used FA and MD. In this study, AD was the parameter with the fewest abnormalities, followed by MD, RD and FA respectively. Considering the limitations, these findings may be related with an active inflammatory process and are in line with pathological investigations of surgical patients (Crespel et al., 2002).

The present whole brain voxel-wise and TBSS results indicated the presence of abnormalities in $80 \%$ of cases in the superior and inferior longitudinal, inferior fronto-occipital and uncinate fasciculi. These white matter fasciculi have a diffuse intra hemispheric localization extending in the axial orientation of the brain. Our findings demonstrate a massive involvement of the temporal lobe white matter with abnormalities in the intricate network of fasciculi that run through this region. The TSA results further revealed major abnormalities in temporal stem topography encompassing the inferior fronto-occipital, inferior longitudinal and uncinate fasciculi in $90 \%$ of cases. Prior research investigating white matter connectivity in MTLE patients has suggested that differences in resections of this circuitry may contribute to different surgical outcomes (Bonilha et al., 2013).
There are investigations suggesting that patients with HS have more widespread abnormalities than patients with a normal MRI (Scanlon et al., 2013). Indeed, patients with left HS are also predisposed to having more diffuse abnormalities (Ahmadi et al., 2009). However, these findings are not uniform (Rodriguez-Cruces and Concha, 2015). The patients investigated here were heterogeneous. Patients had mainly unilateral HS, but bilateral patients were also included. In our study, comparisons according to HS side reveled broad abnormalities in left MTLE.

In patients with MTLE, white matter abnormalities have been localized to the temporal and extra-temporal regions with a centrifugal pattern (Gross, 2011; Otte et al., 2012). Astrogliosis and microstructure rearrangement near the focus and postictal vasogenic edema distally may be related to this pattern (Concha et al., 2012). White matter abnormalities may be observed in up to $63 \%$ of patients submitted to surgical treatment (Kasper et al., 1999; Rodriguez-Cruces and Concha, 2015). The main histological abnormalities described are axonal and myelinic abnormalities, heterotopic neurons in the white matter, blurring of the gray-white matter boundary and white matter gliosis. Reduced axonal density is consistently reported, and the increased extra-cellular space explains the increment of MD and reduction of FA observed in previous investigations (Rodriguez-Cruces and Concha, 2015).

It remains unclear which methodology of voxel-based analysis is the best to evaluate patients with MTLE. Two investigations compared voxel-based analysis using SPM versus TBSS. In one study in which FA and MD of 33 patients with MTLE were investigated, Focke et al. (2008) found widespread abnormalities in the temporal lobe mainly ipsilateral to the HS. Similar to the present study, they found that TBSS was more sensitive to changes for white matter abnormalities. Subsequently, in a study examining FA and ellipsoidal area ratio in 19 patients with MTLE, Afzali et al. (2011) found that TBSS abnormalities were most prominent in the temporal lobe and parahippocampal gyrus. Meanwhile, SPM analysis disclosed abnormalities mainly in the temporal lobes, corpus callosum and fornix. The authors concluded that TBSS was relatively more localized and that ellipsoidal area ratio was more sensitive to white matter abnormalities than FA. Both investigations used conventional registration of the images.

Registration is a critical issue in voxel-based analysis. Without careful performance and checking of this procedure, false positive results may occur (Bookstein, 2001; Schwarz et al., 2014). In this study, registration was performed with the use of the tensor map as is recommended for DTI (Wang et al., 2011). This approach uses full tensor information to drive a more precise alignment (Keihaninejad et al., 2013).

The restriction of our whole brain voxel-wise analysis results to the temporal lobe may be related to the use of Family-Wise Error correction in the statistical analysis. It has been suggested that this threshold may be too rigorous and omit true positive results (Henley et al., 2010). Although a similar correction was applied in our TBSS, the different statistical approach used for the TBSS likely minimized this effect (Winkler et al., 2014), yielding the identification of more regionally extensive abnormalities, especially for FA. These findings are in agreement with the notion that MTLE patients suffer from extra-temporal abnormalities.

One drawback of this investigation was the number of diffusion directions acquired. A large number of sampling orientations is recommended to avoid biases (Jones, 2004). However, for this investigation, this impact was reduced because the main objective was not to develop detailed representation of structural abnormalities. This study was designed to access variation across the main diffusion parameters and to compare two techniques of voxelbased analysis when applied to DTI images acquired using the same protocol. 


\section{Conclusion}

The findings here lend support that different approaches may contribute to the current knowledge of the mechanisms behind MTLE. The use of all diffusion parameters and distinct techniques is advised because they may show additional findings.

\section{Conflicts of interest}

None.

\section{Acknowledgement}

Supported by grants number 2011/02961-2 and 2016/17914-3, São Paulo Research Foundation (FAPESP).

\section{References}

Abe, O., Takao, H., Gonoi, W., et al., 2010. Voxel-based analysis of the diffusion tensor. Neuroradiology 52, 699-710.

Afzali, M., Soltanian-Zadeh, H., Elisevich, K.V., 2011. Tract based spatial statistica analysis and voxel based morphometry of diffusion indices in temporal lobe epilepsy. Comput. Biol. Med. 41, 1082-1091.

Ahmadi, M.E., Hagler Jr., D.J., McDonald, C.R., et al., 2009. Side matters: diffusion tensor imaging tractography in left and right temporal lobe epilepsy. AJNR Am. J. Neuroradiol. 30, 1740-1747.

Alexander, A.L., Lee, J.E., Lazar, M., Field, A.S., 2007. Diffusion tensor imaging of the brain. Neurotherapeutics 4, 316-329.

Ashburner, J., Friston, K.J., 2000. Voxel-based morphometry-the methods. Neuroimage 11, 805-821.

Bach, M., Laun, F.B., Leemans, A., et al., 2014. Methodological considerations on tract-based spatial statistics (TBSS). Neuroimage 100, 358-369.

Behrens, T.E., Woolrich, M.W., Jenkinson, M., et al., 2003. Characterization and propagation of uncertainty in diffusion-weighted MR imaging. Magn. Reson. Med. 50, 1077-1088.

Berg, A.T., Berkovic, S.F., Brodie, M.J., et al., 2010. Revised terminology and concepts for organization of seizures and epilepsies: report of the ILAE Commission on Classification and Terminology, 2005-2009. Epilepsia 51, 676-685.

Bonilha, L., Helpern, J.A., Sainju, R., et al., 2013. Presurgical connectome and postsurgical seizure control in temporal lobe epilepsy. Neurology 81 , 1704-1710.

Bookstein, F.L., 2001. Voxel-based morphometry should not be used with imperfectly registered images. Neuroimage 14, 1454-1462.

Commission on Classification and Terminology of the International League Against Epilepsy, 1989. Proposal for revised classification of epilepsies and epileptic syndromes. Epilepsia 30, 389-399.

Concha, L., Kim, H., Bernasconi, A., Bernhardt, B.C., Bernasconi, N., 2012. Spatial patterns of water diffusion along white matter tracts in temporal lobe epilepsy. Neurology 79, 455-462.

Crespel, A., Coubes, P., Rousset, M.C., et al., 2002. Inflammatory reactions in human medial temporal lobe epilepsy with hippocampal sclerosis. Brain Res. 952, 159-169.

Fischl, B., Salat, D.H., Busa, E., et al., 2002. Whole brain segmentation: automated labeling of neuroanatomical structures in the human brain. Neuron 33 , 341-355.
Fischl, B., van der Kouwe, A., Destrieux, C., et al., 2004. Automatically parcellating the human cerebral cortex. Cereb. Cortex 14, 11-22.

Focke, N.K., Yogarajah, M., Bonelli, S.B., et al., 2008. Voxel-based diffusion tensor imaging in patients with mesial temporal lobe epilepsy and hippocampal sclerosis. Neuroimage 40, 728-737.

Gross, D.W., 2011. Diffusion tensor imaging in temporal lobe epilepsy. Epilepsia 52 (Suppl. 4), 32-34

Henley, S.M., Ridgway, G.R., Scahill, R.I., et al., 2010. Pitfalls in the use of voxel-based morphometry as a biomarker: examples from huntington disease. AJNR Am. J. Neuroradiol. 31, 711-719.

Jenkinson, M., Beckmann, C.F., Behrens, T.E., Woolrich, M.W., Smith, S.M., 2012. Fsl. Neuroimage 62, 782-790.

Jones, D.K., Knösche, T.R., Turner, R., 2013. White matter integrity, fiber count, and other fallacies: the do's and don'ts of diffusion MRI. Neuroimage 73, 239-254.

Jones, D.K., 2004. The effect of gradient sampling schemes on measures derived from diffusion tensor MRI: a Monte Carlo study. Magn. Reson. Med. 51, 807-815.

Kasper, B.S., Stefan, H., Buchfelder, M., Paulus, W., 1999. Temporal lobe microdysgenesis in epilepsy versus control brains. J. Neuropathol. Exp. Neurol. 58, 22-28.

Keihaninejad, S., Zhang, H., Ryan, N.S., et al., 2013. An unbiased longitudinal analysis framework for tracking white matter changes using diffusion tensor imaging with application to Alzheimer's disease. Neuroimage 72, 153-163.

Mori, S., 2005. MRI Atlas of Human White Matter, 1 st ed. Elsevier, Amsterdam; Boston.

Otte, W.M., van Eijsden, P., Sander, J.W., Duncan, J.S., Dijkhuizen, R.M., Braun, K.P., 2012. A metaanalysis of white matter changes in temporal lobe epilepsy as studied with diffusion tensor imaging. Epilepsia 53, 659-667.

Rodriguez-Cruces, R., Concha, L., 2015. White matter in temporal lobe epilepsy: clinicopathological correlates of water diffusion abnormalities. Quant. Imaging Med. Surg. 5, 264-278.

Rorden, C., Brett, M., 2000. Stereotaxic display of brain lesions. Behav. Neurol. 12, 191200.

Scanlon, C., Mueller, S.G., Cheong, I., Hartig, M., Weiner, M.W., Laxer, K.D., 2013. Grey and white matter abnormalities in temporal lobe epilepsy with and without mesial temporal sclerosis. J. Neurol. 260, 2320-2329.

Schwarz, C.G., Reid, R.I., Gunter, J.L., et al., 2014. Improved DTI registration allows voxel-based analysis that outperforms tract-based spatial statistics. Neuroimage 94, 65-78.

Smith, S.M., Jenkinson, M., Johansen-Berg, H., et al., 2006. Tract-based spatial statistics: voxelwise analysis of multi-subject diffusion data. Neuroimage 31 1487-1505.

Smith, S.M., 2002. Fast robust automated brain extraction. Hum. Brain Mapp. 17, 143-155.

Talairach, J., Tournoux, P., 1988. Co-planar Stereotaxic Atlas of the Human Brain : 3-dimensional Proportional System: an Approach to Cerebral Imaging. Thieme Medical Publishers, Stuttgart; New York: G. Thieme; New York.

Wang, Y., Gupta, A., Liu, Z., et al., 2011. DTI registration in atlas based fiber analysis of infantile Krabbe disease. Neuroimage 55, 1577-1586.

Winkler, A.M., Ridgway, G.R., Webster, M.A., Smith, S.M., Nichols, T.E., 2014 Permutation inference for the general linear model. Neuroimage 92, 381-397.

Yasuda, C.L., Betting, L.E., Cendes, F., 2010. Voxel-based morphometry and epilepsy. Expert Rev. Neurother. 10, 975-984.

Yushkevich, P.A., Zhang, H., Simon, T.J., Gee, J.C., 2008. Structure-specific statistical mapping of white matter tracts. Neuroimage 41, 448-461.

Zhang, H., Yushkevich, P., Rueckert, D., Gee, J., 2010. A computational white matter atlas for aging with surface-based representation of fasciculi. In: Fischer, B., Dawant, B., Lorenz, C. (Eds.), Biomedical Image Registration. Springer, Berlin Heidelberg, pp. 83-90.

Zhang, S., Peng, H., Dawe, R.J., Arfanakis, K., 2011. Enhanced ICBM diffusion tensor template of the human brain. Neuroimage 54, 974-984. 